\title{
Viability of the Critically Endangered eastern Taiwan Strait population of Indo-Pacific humpback dolphins Sousa chinensis
}

\author{
Claryana C. Araújo ${ }^{1,2, *}$, John Y. Wang ${ }^{3,4}$, Samuel K. Hung ${ }^{5}$, Bradley N. White ${ }^{3}$, \\ Daniel Brito ${ }^{1}$ \\ ${ }^{1}$ Applied Ecology and Conservation Lab, Departamento de Ecologia, Instituto de Ciências Biológicas, \\ Universidade Federal de Goiás, 74001-970, Goiânia, Goiás, Brazil \\ ${ }^{2}$ Projeto Boto Araguaia, 74310-040, Goiânia, Goiás, Brazil \\ ${ }^{3}$ Department of Biology, Trent University, Peterborough, Ontario K9J 7B8, Canada \\ ${ }^{4}$ CetAsia Research Group, Thornhill, Ontario L4J 7X1, Canada \\ ${ }^{5}$ Hong Kong Cetacean Research Project, Lam Tin, Kowloon, Hong Kong
}

\begin{abstract}
The population of humpback dolphins Sousa chinensis that resides in the eastern Taiwan Strait (ETS) is geographically and genetically isolated from populations inhabiting the coastal waters of mainland China and appears to be facing unsustainable levels of human threats. Using VORTEX 9.99b, we modeled the viability of this Critically Endangered population under the present conditions as well as under realistic additional threat scenarios. We examined 6 different levels of bycatch mortality, 3 scenarios of habitat loss/degradation, and scenarios in which these threats were experienced together. Under the baseline (present) scenario, the population exhibited a decreasing growth rate and was predicted to be smaller than the initial population size in more than $76 \%$ of all model runs. In all scenarios with additional threats, the proportion of model runs in which population size was smaller than the initial size varied from 77.1 to $92.6 \%$. Over the short term, fisheries-related mortality appears to have a more obvious impact on the population's trajectory than habitat loss/degradation. Even minimal increases in mortality from the current baseline levels will increase the probability of extinction of this population. Due to the fragile situation of ETS humpback dolphins, mitigation actions to reduce the current threats to this population are needed immediately.
\end{abstract}

KEY WORDS: Population modeling $\cdot$ Cetacean $\cdot$ Conservation $\cdot$ Marine mammal Resale or republication not permitted without written consent of the publisher

\section{INTRODUCTION}

The population of Indo-Pacific humpback dolphins Sousa chinensis that resides in the eastern Taiwan Strait (ETS) along the western coast of Taiwan was only recently discovered (Wang et al. 2004). It is geographically and genetically isolated from adjacent populations found in the coastal waters of mainland China (Wang et al. 2008). This population inhabits a small area of coastal shallow waters (about $600 \mathrm{~km}^{2}$ ), within $3 \mathrm{~km}$ from the shore and generally in water less than 30 m deep (Wang et al. 2007a,b). Like other populations of the species in Chinese waters (Jefferson 2000, Jefferson \& Karczmarski 2001), the ETS population also seems to be dependent upon the estuaries of major river systems (Wang et al. 2004, 2007a, Ross et al. 2010).

Unfortunately, the waters in which this small population lives are used extensively by humans, and human activities are also intensive in adjoining coastal areas (Wang et al. 2007a, Ross et al. 2010, Dungan et al. 2011). As a consequence, this population has 
been, and continues to be, severely impacted by 5 main threats: habitat loss, anthropogenic noise, pollution, reduction of freshwater inputs, and fisheries bycatch (see Wang et al. 2007a,b, Reeves et al. 2008, Ross et al. 2010, Dungan et al. 2011). For example, several proposals for more large-scale industrial development that require massive land reclamation (for some examples, see Wang et al. 2007b) signal that habitat loss and degradation are likely to worsen in the near future, thus compromising habitat quantity and quality for humpback dolphins. Regarding the impacts of fisheries, field observations have shown that more than $30 \%$ of the ETS dolphins exhibit serious scars/wounds that are most likely fisheries-related (Slooten et al. 2013).

An initial preliminary abundance estimate based on line-transect data has shown that this population is small, comprising only 99 ind. (CV $=51.6 \%$; Wang et al. 2007a). Because of the small population size, and inferred decline due to ongoing threats (Wang et al. 2007b, Ross et al. 2010, Dungan et al. 2011), this population of humpback dolphins was listed as Critically Endangered on the IUCN Red List of Threatened Species (Reeves et al. 2008). Recently, mark-recapture analyses of photo-identification data generated more precise abundance estimates, with a maximum annual point estimate of only 74 ind. (Wang et al. 2012), suggesting that the population is smaller than previously indicated.

Small populations are more vulnerable to stochastic processes or events that may increase their chances of ultimately being driven into an extinction vortex (Caughley 1994, Lacy 2000). Therefore, understanding how current threats affect the viability of populations (especially small ones such as the ETS population) is of great importance for guiding conservation strategies. In this context, population viability analysis is a promising tool for examining the relative risks posed by different threats to the persistence of small populations, and evaluating the effectiveness of possible management strategies and actions for their recovery (Akçakaya 2000, Beissinger \& McCullough 2002, Morris \& Doak 2002).

The only analysis of population viability for $S$. chinensis to date has been for the animals of the Pearl River Estuary, including Hong Kong's waters (Huang et al. 2012). This analysis was performed using demographic information obtained from carcasses found stranded on the shores or floating at sea. However, information obtained from stranded carcasses may be a biased representation of the living population (Wang 2009), so information such as mortality rate (and therefore survivorship) and other demographic pa- rameters may not be accurately reflected. Markrecapture analysis of photo-identification data to estimate demographic parameters directly from the living population is likely to provide a more accurate representation of the population. However, such information is still unavailable for many cetacean species and populations because mark-recapture analyses require long-term data collection, tend to be resource- and time-intensive, and the behaviour of some species is not conducive to the tracking of individuals over several years. Fortunately, the ETS population of humpback dolphins possesses characteristics that make it an ideal population for mark-recapture analyses (see Wang et al. 2012). The recent precise abundance and first survivorship estimates presented by Wang et al. (2012) provided information allowing a more reliable examination of the extinction risks of this population under various scenarios. The objective of the present study was to model the viability of the ETS $S$. chinensis population and to understand how different additional realistic threat scenarios of recognized human activities would affect the dynamics of this highly endangered population (Wang et al. 2007a,b, Reeves et al. 2008, Ross et al. 2010, Dungan et al. 2011) .

\section{METHODS}

\section{Overview}

The 5 main recognized threats affecting this population can be grouped into 2 classes for modeling purposes: removals (i.e. due to fisheries) and habitat loss/degradation (by anthropogenic noise, pollution, reduction of freshwater inputs). All of these threats may be found along the entire ETS dolphin population habitat, which comprises about $200 \mathrm{~km}$ (linear distance) of coastal, inshore waters along western Taiwan (Fig. 1).

Reliable information on coastal fisheries along western Taiwan is sparse even though fisheries activities are intensive in these waters. Most of the fishing vessels employ gillnets (mainly trammel nets) or trawl nets (Ross et al. 2010). Bottom trawls are restricted to waters farther than 3 nautical miles $(\sim 5.6 \mathrm{~km})$ from shore, but continuing illegal inshore trawling with minimal enforcement has resulted in overlap with much of the ETS dolphin habitat (J. Y. Wang unpublished data). In contrast, trammel nets are plentiful and are used with no restrictions (Slooten et al. 2013). There is no direct estimate of bycatch levels (or even fishing effort), but gillnets are used everywhere within the study area (Slooten et al. 


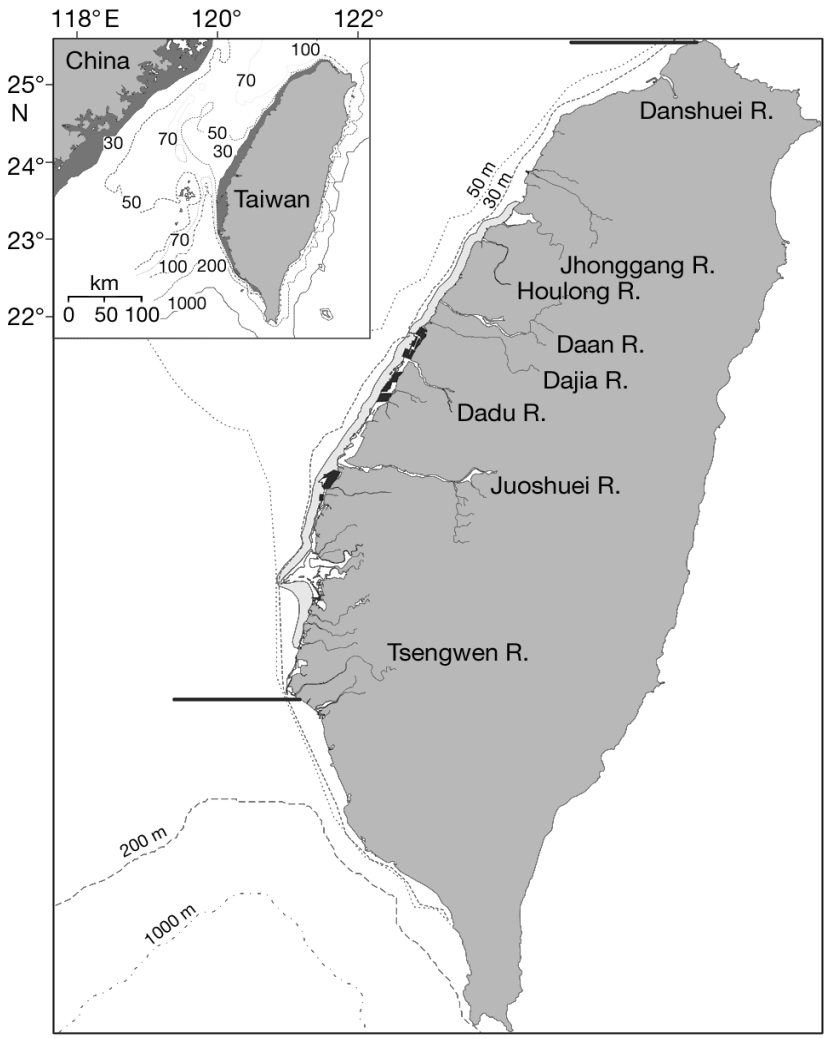

Fig. 1. Eastern Taiwan Strait (ETS) between mainland China and Taiwan (inset) and the habitat of the ETS humpback dolphin Sousa chinensis population. The confirmed habitat is indicated by the light grey shaded area and the suitable habitat (following Ross et al. 2010) by bold horizontal lines. Dark grey shading on inset map shows water $<30 \mathrm{~m}$ deep

2013). Even the bycatch capture of a single individual from this small population each year would be unsustainable based on a calculation of the potential biological removal (PBR) level, which was 1 individual every 7 years (Slooten et al. 2013).

Besides the direct loss of habitat through land reclamation for large development projects, the qual- ity of ETS Sousa chinensis habitat is being compromised by several sources of pollution, both marine based (e.g. from shipyards) and land based (e.g. industrial effluents; Wang et al. 2007b). Along Taiwan's west coast, 623 factories are located within $1 \mathrm{~km}$ of the shoreline (Wang et al. 2007b). The habitat quality of this population is further compromised by intense anthropogenic noise from marine and coastal construction, vessels, military exercises, and seismic research (Ross et al. 2010). Thus, the effects of mortality due to fisheries-related activities (bycatch) and habitat loss/degradation were the threat scenarios examined in this study.

\section{Population viability analysis (PVA)}

PVA was performed using VORTEX 9.99b, a Monte Carlo simulation package designed to model the effects of deterministic factors and demographic, environmental, and genetic stochasticity on population dynamics (Lacy et al. 2009).

Demographic parameters (e.g. mortality rates, survivorship, abundance) used to model the ETS humpback dolphin population were obtained from published population ecology studies conducted on this species (Table 1). The values for the demographic parameters used in the VORTEX analyses are presented in Table 1. Extinction was defined in this study to have been reached when only 1 sex remained.

For the age of first reproduction (from the population of the Pearl River Estuary, PRE), the middle value of age estimates was used for precautionary reasons. Because the middle value for the female estimates was not an integer, we rounded the value upward. The value for the maximum age of reproduction was based on a 5 yr calving interval, 38 yr of

Table 1. Summary of demographic parameters used as input data in the modeling of the viability of the eastern Taiwan Strait population of humpback dolphins Sousa chinensis. Standard deviations due to environmental variation are shown in parentheses

\begin{tabular}{|lcl|}
\hline Parameter & Value & Reference \\
\hline Mating system & Polygynous & T. A. Jefferson (pers. comm.) \\
Sex ratio at birth (\% males) & 50 & T. A. Jefferson (pers. comm.) \\
First age of reproduction (females/males) & $10 / 13 \mathrm{yr}$ & Jefferson et al. (2012) \\
Max. age of reproduction & $35 \mathrm{yr}$ & Based on Jefferson et al. (2012) (see 'Methods') \\
Max. no. of broods yr ${ }^{-1}$ & 1 & Jefferson \& Karczmarski (2001) \\
Max. no. of progeny per brood yr ${ }^{-1}$ & 1 & Jefferson \& Karczmarski (2001) \\
Carrying capacity (K) & 250 & Present study; see 'Methods' \\
\% adult females breeding & $10( \pm 1)$ & J. Y. Wang (unpublished data) \\
Mortality rate (\%) & $1.5( \pm 0.022)$ & Wang et al. (2012) (see 'Methods') \\
Initial population size & 74 & Wang et al. (2012) \\
\hline
\end{tabular}


maximum longevity, and considering a senescence period for the species (Jefferson et al. 2012). Assuming the age of first reproduction for females is $10 \mathrm{yr}$ and the calving interval is $5 \mathrm{yr}$, the last reproductive event would occur at $35 \mathrm{yr}$ because the maximum longevity of $38 \mathrm{yr}$ would not allow another calving event. Thus, the remaining years after 35 would represent senescence; field observations appear to support female reproductive senescence in this population (J. Y. Wang unpublished data). The percentage of breeding females was calculated based on the information of the observed number of females giving birth each year (varying between 0 and 3 , but in most years, 2 neonates are observed in the population; J. Y. Wang unpublished data) and $60 \%$ of the population being mature based on Jefferson (2000). Assuming a 1:1 sex ratio in a population of 74 ind. (Wang et al. 2012), 37 would be females, of which 22 ( $60 \%$ ) would be sexually mature. Thus, the observed presence of 2 neonates per year would indicate that on average, $9.1 \%$ of the females were breeding each year. To simplify the calculations, a baseline value of $10 \%$ was used. The mortality rate $(M)$ was based on the recent estimate of survivorship ( $\phi$ ) for this population of $0.985 \pm 0.018$ (SE) (Wang et al. 2012), which translated to a mortality rate of $0.015(M=1-\phi)$ or $1.5 \pm 0.15 \%$ (SD). The SD was calculated by:

$$
\mathrm{SE}=\frac{\mathrm{SD}}{\sqrt{\mathrm{N}}}
$$

and the environmental variation (EV) of this parameter $(p)$ was calculated based on the total variation (TV) and demographic variation (DV) as follows: $\mathrm{EV}=\mathrm{TV}-\mathrm{DV}$, where $\mathrm{TV}$ is $\mathrm{SD}^{2}$ and $\mathrm{DV}=p(1-p) /$ $(\mathrm{N}-1)$.

For all simulations, 1000 iterations were performed for a time scale of $100 \mathrm{yr}$. This time frame was selected as it is in accordance with many conventions, standards, and practices for species conservation (e.g. IUCN Red List), but for long-lived species, a longer time frame may also be reasonable. The $100 \mathrm{yr}$ time frame also seemed reasonable from a management perspective. The baseline scenario (which represents the current level of threats and a lack of conservation actions) simulated a population of 74 ind. with no additional threats. Based on the current low numbers of ETS humpback dolphins, state of the available habitat, numerous anthropogenic threats, and the fact that almost no conservation measures exist to reduce these threats, it was reasonable to infer that the population is declining (Reeves et al. 2008) and therefore must be below carrying capacity. Thus, the carrying capacity $\left(\mathrm{K}_{;}\right.$number of individuals) for the baseline was set higher than the current highest point estimate of abundance (Wang et al. 2012) at 250 ind., which was the middle value of $\mathrm{K}$ estimated using 2 different methods and from information obtained from the PRE and the ETS humpback dolphin populations. One estimate of $\mathrm{K}$ was calculated using the mean density estimate (36.95 per $100 \mathrm{~km}^{2}$ ) for the PRE population (data from Jefferson 2000) and applied to the entire confirmed habitat of the ETS population (about $630 \mathrm{~km}^{2}$ based on information in Ross et al. 2010), which gave an estimated $\mathrm{K}$ of 233. For the ETS population, we used an estimated K of 266, which was the $95 \%$ upper confidence limit for an abundance estimate that was generated from line-transect analysis (Wang et al. 2007a).

\section{Modeling scenarios}

To examine the impacts of additional bycatch on the ETS humpback dolphin population, 6 different levels of harvest (= mortality) scenarios were modeled: (1) 1 adult female killed every 7 yr, (2) 1 adult female killed every year, (3) 1 adult male killed every 7 yr, (4) 1 adult male killed every year, (5) 1 adult female and 1 male killed every $7 \mathrm{yr}$, and (6) 1 adult female and 1 male killed every year. The levels of bycatch were chosen based on preliminary information from government reports and field data (Wang et al. 2007b, Slooten et al. 2013). The $7 \mathrm{yr}$ period was based on the PBR calculations that were performed by Slooten et al. (2013), who found that the removal of even 1 individual every $7 \mathrm{yr}$ exceeded the PBR for this population.

The remaining threats can all impact the ETS S. chinensis directly (e.g. habitat loss) and indirectly through degradation and thus reduce the carrying capacity of the dolphin habitat. Therefore, the effects of continued habitat loss and degradation were modeled as a decrease in the carrying capacity of the study area. We modeled 3 scenarios wherein carrying capacity was reduced by 5,10 , and $20 \%$. These carrying capacity reduction values for the ETS roughly reflected the sizes of proposed coastal development projects. For example, the 4000 ha Kuokuang Petrochemical complex that was cancelled in 2011 would have represented about 6 to $7 \%$ of the confirmed habitat of the ETS population, while the largest plans for future coastal development approached $20 \%$ (land reclamation data from Wang et al. 2007a). Continuing gradual degradation of the habitat was not considered due to the complexity of 
such simulations so the scenarios presented would represent simplistic but under-estimated reductions in carrying capacity. We also constructed a set of scenarios where bycatch and habitat loss/degradation were realized together.

\section{Incorporating uncertainty and sensitivity analysis}

To explicitly incorporate uncertainty in estimates of model parameters (e.g. Slooten et al. 2000), we included a function in which a value was selected at random from a distribution for each iteration of the simulations. For mortality rates, the values were sampled from a normal distribution with a mean of $1.5 \%$ and standard deviation of 0.15 (see 'Population viability analysis (PVA)'). For the parameter of percentage of breeding females, a uniform distribution from 5 to $14 \%$ was chosen with a standard deviation of $1 \%$ (see below).

To better understand which parameters would most affect population dynamics, a series of sensitivity analyses were performed. Alternative fixed values (lower and higher than the baseline scenario) were tested for age of first reproduction, maximum age of reproduction, and percentage of breeding females (Table 2). The range of values for percentage

Table 2. Alternative fixed values (lower and higher than the baseline scenario) used in the sensitivity analysis for eastern Taiwan Strait humpback dolphins Sousa chinensis. For details, see 'Methods: Incorporating uncertainty and sensitivity analysis'

\begin{tabular}{|lcc|}
\hline \multirow{2}{*}{ Parameter } & \multicolumn{2}{c|}{ Values } \\
\cline { 2 - 3 } & Lower & Higher \\
\hline $\begin{array}{l}\text { First age (yr) of reproduction } \\
\text { (females/males) }\end{array}$ & $8 / 10$ & $12 / 15$ \\
$\begin{array}{l}\text { Max. age (yr) of reproduction } \\
\% \text { breeding females }\end{array}$ & 30 & 40 \\
\hline
\end{tabular}

of breeding females was based on the lowest and highest number of neonates observed per year (from 1 to 3 and not considering years when no neonates were observed). Thus, the lowest and highest values were considered as $5 \%$ and $14 \%$; both values were rounded up for more optimistic scenarios. All sensitivity analyses were conducted with 1000 iterations and over $100 \mathrm{yr}$.

\section{RESULTS}

From the modeling, the generation times were 22.24 and 23.8 yr for females and males, respectively. Thus, the time-span of our simulations (100 yr) represented about 5 generations for the ETS humpback dolphin population. The generation time values are similar to those estimated by Taylor et al. (2007) for Sousa chinensis as well as for other species of small cetaceans such as pantropical spotted dolphin Stenella attenuata and common bottlenose dolphin Tursiops truncatus. Under the baseline scenario which included the current levels of threats, the population exhibited a gradual decreasing trend. In more than $76 \%$ of all model runs, the population size was predicted to be smaller than the initial population size, and in about $66 \%$, the population size was $\leq 1$ ind.

In all scenarios that included additional fisheriesrelated mortality, the population decline was more pronounced; the proportion of model runs resulting in the population size being less than the initial size varied from 77.1 to $92.6 \%$ (Fig. 2). The scenario wherein 1 additional female was removed annually resulted in a high proportion of model runs $(91.7 \%)$ in which the population size decreased. For the annual removal of 1 additional male, this proportion was much lower $(78.4 \%)$. Thus, the influence on the probability of extinction seemed to be biased towards females, with a greatly increased probability of extinction in scenarios where additional females are removed from the population (Table 3).

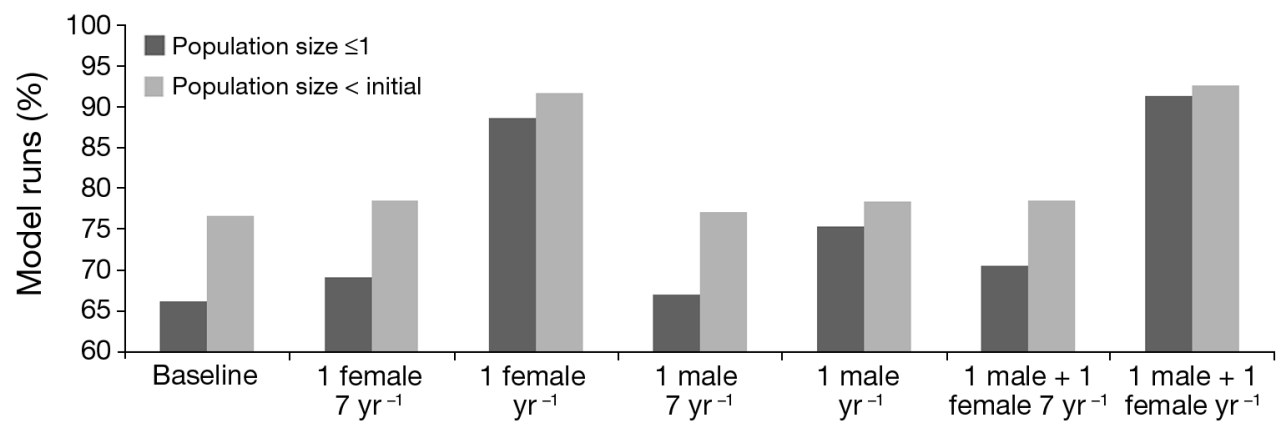

Fig. 2. Percentage of model runs for 2 different population size scenarios for eastern Taiwan Strait humpback dolphins Sousa chinensis under 6 different scenarios of fishery removal. See Table 3 for a description of the scenarios 
The ETS humpback dolphin population also exhibited a population decline for all 3 scenarios of habitat loss/degradation, and the proportion of model runs that resulted in a population size less than the initial or extinction threshold (i.e. $\leq 1$ ind.) was similar across the 3 scenarios (Fig. 3, Table 3).

Although the modeling of single-threat scenarios is useful for understanding the relative impacts of individual threats on population persistence, the real situation is often more complex. To better understand the persistence of this small population, which is experiencing multiple threats concurrently, the effects of both bycatch and habitat loss/degradation were

Table 3. Scenarios of bycatch mortality ('harvest') and habitat loss/degradation for eastern Taiwan Strait humpback dolphins Sousa chinensis and percentage of model runs (1000 iterations) for which the population size was less than the initial population size $\left(\mathrm{N}_{\mathrm{i}}=74\right)$ and the extinction threshold $(\leq 1$ ind. remaining). The harvest scenarios were (1) 1 adult female killed every $7 \mathrm{yr}$, (2) 1 adult female killed every year, (3) 1 adult male killed every 7 yr, (4) 1 adult male killed every year, (5) 1 adult female and 1 male killed every 7 yr, and (6) 1 adult female and 1 male killed every year. Carrying capacity $(\mathrm{K})$ was reduced by 5,10 , or $20 \%$

\begin{tabular}{|c|c|c|}
\hline \multirow[t]{2}{*}{ Scenario } & \multicolumn{2}{|c|}{ Percentage of models run } \\
\hline & $\begin{array}{c}\text { Population } \\
\text { size < initial }\end{array}$ & $\begin{array}{l}\text { Population } \\
\text { size } \leq 1\end{array}$ \\
\hline Baseline & 76.6 & 66.2 \\
\hline Harvest 1 & 78.5 & 69.1 \\
\hline Harvest 2 & 91.7 & 88.6 \\
\hline Harvest 3 & 77.1 & 67 \\
\hline Harvest 4 & 78.4 & 75.4 \\
\hline Harvest 5 & 78.5 & 70.5 \\
\hline Harvest 6 & 92.6 & 91.3 \\
\hline K $5 \%$ & 76.3 & 66.2 \\
\hline K $10 \%$ & 76.8 & 65.1 \\
\hline K $20 \%$ & 76.1 & 65.3 \\
\hline
\end{tabular}

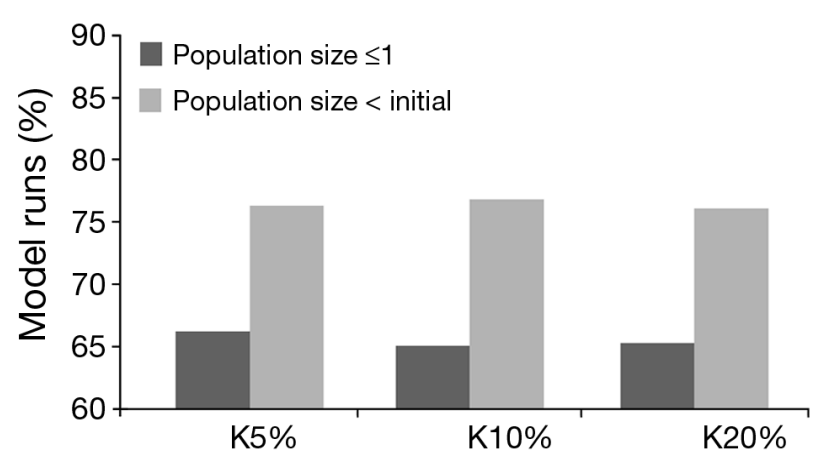

Fig. 3. Percentage of model runs for 2 different population size scenarios for eastern Taiwan Strait population of humpback dolphins Sousa chinensis under 3 different scenarios of habitat degradation, whereby carrying capacity (K) was reduced by 5,10 , or $20 \%$ considered together. For the combined simulations, the decline in population size did not differ greatly among the different scenarios of habitat loss/degradation $(5,10$, and $20 \%)$ under the same level of harvest (Table 4).

Based on the sensitivity analyses, changes in the values for first and maximum ages of reproduction did not noticeably alter population growth rate. In contrast, the population growth rate was most sensitive to changes in the percentage of breeding females (Fig. 4). A $4 \%$ increase in breeding females

Table 4. Scenarios of the combined impacts of bycatch mortality ('harvest') and habitat loss/degradation and the percentage of model runs (1000 iterations) for which the population size was less than the initial population size $\left(\mathrm{N}_{\mathrm{i}}=74\right)$ and the extinction threshold ( $\leq 1$ ind. remaining). See Table 3

for a description of the scenarios. K: carrying capacity

\begin{tabular}{|lcc|}
\hline Scenario & Percentage of models run \\
\cline { 2 - 3 } & $\begin{array}{c}\text { Population } \\
\text { size < initial }\end{array}$ & $\begin{array}{c}\text { Population } \\
\text { size } \leq 1\end{array}$ \\
\hline Harvest 1, K 5\% & 78.9 & 69.8 \\
Harvest 2, K 5\% & 78.4 & 69.3 \\
Harvest 3, K 5\% & 78.8 & 69.1 \\
Harvest 4, K 5\% & 92 & 89.3 \\
Harvest 5, K 5\% & 92.9 & 90.4 \\
Harvest 6, K 5\% & 91.6 & 89.3 \\
Harvest 1, K 10\% & 77.3 & 67.5 \\
Harvest 2, K 10\% & 76.6 & 68.1 \\
Harvest 3, K 10\% & 76.9 & 68 \\
Harvest 4, K 10\% & 78.4 & 76 \\
Harvest 5, K 10\% & 78.3 & 75.1 \\
Harvest 6, K 10\% & 78.7 & 76.1 \\
Harvest 1, K 20\% & 79 & 70.3 \\
Harvest 2, K 20\% & 78.5 & 71.4 \\
Harvest 3, K 20\% & 78.8 & 69.1 \\
Harvest 4, K 20\% & 92.5 & 91.2 \\
Harvest 5, K 20\% & 92.5 & 91.2 \\
Harvest 6, K 20\% & 92.3 & 91.5 \\
\hline
\end{tabular}

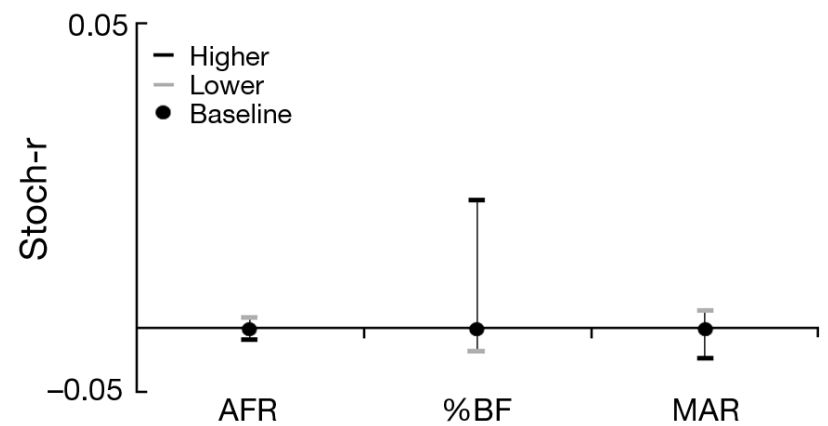

Fig. 4. Sensitivity analyses of age of first reproduction (AFR), maximum age of reproduction (MAR), and percentage of breeding females (\%BF) for eastern Taiwan Strait humpback dolphins Sousa chinensis. Alternative values of the higher and lower scenarios are shown in Table 2. Stoch-r: stochastic growth rate 
(which is equivalent to an additional neonate produced per year for this population) resulted in a large increase in population growth $(\mathrm{r}=0.002)$, whereas a $5 \%$ decrease (which is equivalent to 1 fewer neonate per year) resulted in a decline in the population growth rate $(\mathrm{r}=-0.039)$.

\section{DISCUSSION}

On a global scale, interactions with fisheries represent the main threat to small cetaceans (Read et al. 2006). Bycatch can lead to a direct depletion of cetacean numbers and thus has important demographic consequences, especially for species with small populations (Wade 1998, Reeves et al. 2003, Dawson \& Slooten 2005, Read et al. 2006). Several populations and species are seriously threatened as a result of fisheries-related mortality, such as the vaquita Phocoena sinus (Rojas-Bracho et al. 2006) and Hector's dolphin Cephalorhynchus hectori (Slooten \& Dawson 2010). Bycatch by local fisheries is believed to have been the primary cause of the functional extinction of the baiji Lipotes vexillifer in the Yangtze River of China (Turvey et al. 2007).

Our analyses showed that fishery bycatch is a greater threat to the Critically Endangered population of humpback dolphins in the ETS than the simulated levels of habitat degradation. Furthermore, even a minimal increase in bycatch mortality can greatly increase the probability of extinction for this small population. This is particularly worrisome because there is no formal monitoring of fishing activities or of their impacts on the dolphins, and no actions have been taken to reduce this threat (Wang et al. 2007b).

Fisheries activities in Taiwan have increased dramatically as indicated by a more than $750 \%$ increase in the number of fishing vessels since the 1950s (Huang \& Chuang 2010). Many types of fishing gear are being used in Taiwanese coastal waters, including gillnets (mainly trammel nets along western Taiwan), trawls, and set-nets, and these all represent a threat to the ETS population at some level. However, gillnets and trammel nets overwhelmingly pose the greatest threat to this population (Wang et al. 2007b, Ross et al. 2010, Dungan et al. 2011), especially given the number of nets that are being used and because trawl fishing is banned from the waters within 3 nautical miles from shore (i.e. ETS dolphin habitat) and therefore should pose less of a threat. The ETS dolphins have been observed with scars related to interactions with fisheries, and several individuals have been photographed with entangled lines around their body including a young calf in early June 2012 (J. Y. Wang unpubl. data). In 2009, a stranded dolphin (known since the inception of a catalog of recognizable individuals that has been maintained by the FormosaCetus Research and Conservation Group) exhibited injuries that resulted from gillnet entanglement and likely caused its death (J. Y. Wang unpubl. data).

For small populations, the removal of any individual (especially females in this study) may have a great impact on the dynamics of the population. For example, such removals can seriously disrupt the social organization of small populations and further increase their extinction risks (this was not examined here due to the complexity of such analyses). Our study demonstrates the importance of females in this population. For mammals with a polygynous mating system (such as in this species), where a single male is capable of fertilizing all of the few receptive females available annually, the present results were not too surprising. Also, as shown by Dungan (2011) for the ETS humpback dolphin population, breeding females are important for the social network structure in general. Moreover, based on the sensitivity tests, the percentage of breeding females appeared to be a critical parameter and supported the idea that a higher recruitment rate is necessary to increase the population growth (since the survival rate is high). However, if our analyses included considerations for genetics, then the loss of males would likely carry greater importance for resilience of the population through their contributions to genetic diversity.

Although habitat loss/degradation alone did not increase the population decline compared to the baseline, a more impaired habitat may affect the dynamics of the population by compromising its growth and ultimately its persistence over the long term. It was also interesting to note that it would have been unlikely for the declining population in the model to reach $\mathrm{K}$ in any of the scenarios of habitat change, which meant that changes in $\mathrm{K}$ had little to no effect on the results.

Moreover, habitat degradation (including pollution and diversion of freshwater) in combination with mortality caused by fisheries may have a synergistic effect on population dynamics. Although the synergistic effects of threats on population dynamics may be difficult to quantify and understand, they cannot be neglected (Reeves et al. 2003). Deterioration of habitat quality will likely also contribute to increased natural mortality and decreased offspring production, which may further reduce population size. With 
a smaller population size, the population may become even more susceptible to environmental and genetic stochasticity (Lande 1988).

The effects of habitat loss/degradation were likely underestimated in the present study because the only effect considered was a decrease in carrying capacity. The loss of habitat quality can also affect nutrition, health, survival, and reproductive rates. It has been demonstrated that organochlorines (such as DDT) can cause reproductive dysfunction and increased mortality (from infection) due to the immuno-suppression in marine mammals (e.g. Parsons 2004, Reijnders et al. 2009). The ETS humpback dolphins also appear to be exposed to high levels of contamination, with $68 \%$ of the population predicted to be at risk for immunotoxicity from PCBs (Riehl 2012).

The various proposed industrial development projects that require land reclamation in western Taiwan's coastal waters will reduce the habitat of the ETS humpback dolphins. With a reduction in the habitat available for these dolphins, the impacts of fisheries can be magnified in a small and shrinking habitat. Moreover, the loss of habitat quality and habitat fragmentation could divide the population into smaller isolated groups that can plunge into extinction vortices more rapidly (Brito \& Fernandez 2000) and hamper the dolphins' ability to associate. This could lead to a reduction in survival and/or the reproductive benefits gained by social organization (Dungan 2011).

Our analysis was conservative. Other factors that may also have important impacts on the population were not considered due to the lack of data. For example, information on genetics, more detailed data on the impacts of slow habitat degradation (such as diversion of freshwater), depletion of prey, and pollution are not available or easily quantifiable at present. Also, not considering age-specific survivorship (e. g. juveniles likely having lower survivorship) means that our probability of extinction was underestimated. These data are still being obtained, and when they become available, new models can be simulated in more detail. However, we incorporated parameter uncertainty in the simulations which led to more robust conclusions; thus the lack of precision in some of the parameter estimates should not be reason to delay management actions (e.g. Slooten et al. 2000, Currey et al. 2009). Even with such uncertainties, the ETS population had a high probability of decline and therefore, the present results should not be interpreted more optimistically but rather as an indication that more actions should be taken to ensure the population's persistence.
Comparisons of relative risks are entirely dependent on the variety of values tested. Here, the reported relative effects of different threats (set of values tested) represented plausible levels of impacts based on what is currently known about possible emerging threats. This initial viability analysis for the ETS humpback dolphin population helped to highlight which threats still lack understanding of their effects on the population. More information on the impacts of habitat degradation throughout the ETS humpback dolphin habitat would be useful for further recognizing which and how demographic parameters are affected by these activities. Conducting simulations with such detailed information would further reduce the simplistic and optimistic scenarios and may change the relative risks presented in this study.

Finally, this study presented important findings that can be applied to adaptive management plans such as identifying minimum mortality reductions that would allow the population to increase. Since the gillnet fisheries in Taiwan are mainly artisanal/ subsistence, conducted from small boats and comprised of thousands of fishermen, it would be economically and logistically impossible to implement a monitoring program (e.g. observers on board) with adequate coverage to ensure that a threshold bycatch limit is strictly controlled. Also, there is no method to ensure that females are not being caught. Therefore, there does not appear to be a solution to the fisheries-caused mortality other than a total ban of gillnets within the habitat of the ETS humpback dolphins. Different management measures were explored by Slooten et al. (2013), who suggested alternative, non-harmful fishing gear as a possible solution to gillnet mortalities.

Due to the fragile situation of the ETS humpback dolphins, mitigation actions to reduce the current threats to this population are needed immediately. A reduction in mortality due to bycatch in gillnets is clearly an urgent priority. Additionally, reducing habitat degradation (e.g. pollution and land reclamation) will also certainly contribute to better habitat quality for this population and diminish the likelihood of its extinction.

Acknowledgements. We greatly appreciate the comments of R.J. Currey on earlier drafts of this paper and the valuable suggestions of R. Lacy on the functions for the uncertainty analysis C.C.A. is supported by a PhD scholarship from CAPES - Coordenação de Aperfeiçoamento de Pessoal de Nível Superior (Brazil). D.B.'s research is supported by the CNPq - Conselho Nacional de Desenvolvimento Científico e Tecnológico (Project \#305631/2009-8) (Brazil). 


\section{LITERATURE CITED}

Akçakaya HR (2000) Population viability analyses with demographically and spatially structured models. Ecol Bull 48:23-38

Beissinger SR, McCullough DR (2002) Population viability analysis. University of Chicago Press, Chicago, IL

> Brito D, Fernandez FAS (2000) Metapopulation viability of the marsupial Micoureus demerarae in small Atlantic forest fragments in south-eastern Brazil. Anim Conserv 3:201-209

Caughley G (1994) Directions in conservation biology. J Anim Ecol 63:215-244

Currey RJC, Dawson SM, Slooten E (2009) An approach for regional threat assessment under IUCN Red List criteria that is robust to uncertainty: the Fiordland bottlenose dolphins are critically endangered. Biol Conserv 142:1570-1579

Dawson SM, Slooten E (2005) Management of gillnet bycatch of cetaceans in New Zealand. J Cetacean Res Manag 7:59-64

Dungan SZ (2011) Comparing the social structures of IndoPacific humpback dolphins (Sousa chinensis) from Pearl River Estuary and eastern Taiwan Strait. MSc thesis, Trent University, Peterborough, ON

Dungan SZ, Riehl KN, Wee A, Wang JY (2011) A review of the impacts of anthropogenic activities on the critically endangered eastern Taiwan Strait Indo-Pacific humpback dolphins (Sousa chinensis). J Mar Anim Ecol 4:3-9

Huang HW, Chuang CT (2010) Fishing capacity management in Taiwan: experiences and prospects. Mar Policy 34:70-76

Huang SL, Karczmarski L, Chen J, Zhou R and others (2012) Demography and population trends of the largest population of Indo-Pacific humpback dolphins. Biol Conserv 147:234-242

Jefferson TA (2000) Population biology of the Indo-Pacific hump-backed dolphin in Hong Kong waters. Wildl Monogr 144:1-65

Jefferson TA, Karczmarski L (2001) Sousa chinensis. Mamm Species 655:1-9

- Jefferson TA, Hung SK, Robertson KM, Archer FI (2012) Life history of the Indo-Pacific humpback dolphin in the Pearl River Estuary, southern China. Mar Mamm Sci 28:84-104

Lacy RC (2000) Considering threats to the viability of small populations using individual-based models. Ecol Bull 48: $39-51$

Lacy RC, Borbat M, Pollak JP (2009) VORTEX: a stochastic simulation of the extinction process. Version 9.99. Chicago Zoological Society, Brookfield, IL

- Lande R (1988) Genetics and demography in biological conservation. Science 241:1455-1460

Morris WF, Doak DF (2002) Quantitative conservation biology: theory and practice of population viability analyses. Sinauer Associates, Sunderland, MA

Parsons ECM (2004) The potential impacts of pollution on humpback dolphins with a case study on the Hong Kong population. Aquat Mamm 30:18-37

Read AJ, Drinker P, Northridge S (2006) Bycatch of marine mammals in U.S. and global fisheries. Conserv Biol 20: 163-169

Reeves RR, Smith BD, Crespo E, di Sciara GN, Cetacean Specialist Group (2003) Dolphins, whales, and porpoises: 2003-2010 conservation action plan for the world's cetaceans. IUCN Species Survival Commission, Gland

Reeves RR, Dalebout ML, Jefferson TA, Karczmarski L and others (2008) Sousa chinensis (eastern Taiwan Strait subpopulation). IUCN 2012. Red List of Threatened Species. Version 2012.1. Available at www.iucnredlist.org (accessed on 25 August 2012)

Reijnders PJH, Aguilar A, Borrel A (2009) Pollution and marine mammals. In: Perrin WF, Wursig B, Thewissen JGM (eds) Encyclopedia of marine mammals. Academic Press and Elsevier, San Diego, CA, p 890-898

Riehl KN (2012) Modelling bioaccumulation and pharmacokinetics of polychlorinated biphenyls (PCBs) in toothed whales. MSc thesis, Trent University, Peterborough, ON

Rojas-Bracho L, Reeves RR, Jaramillo-Legorreta A (2006) Conservation of the vaquita Phocoena sinus. Mammal Rev 36:179-216

Ross PS, Dungan SZ, Hung SK, Jefferson TA and others (2010) Averting the baiji syndrome: conserving habitat for critically endangered dolphins in Eastern Taiwan Strait. Aquat Conserv 20:685-694

Slooten E, Dawson SM (2010) Assessing the effectiveness of conservation management decisions: likely effects of new protection measures for Hector's dolphin (Cephalorhynchus hectori). Aquat Conserv 20:334-347

> Slooten E, Fletcher D, Taylor BL (2000) Accounting for uncertainty in risk assessment: case study of Hector's dolphin mortality due to gillnet entanglement. Conserv Biol 14:1264-1270

> Slooten E, Wang JY, Dungan SZ, Forney KA and others (2013) Impacts of fisheries on the Critically Endangered humpback dolphin Sousa chinensis population in the eastern Taiwan Strait. Endang Species Res 22:99-114

Taylor BL, Chivers SJ, Larese J, Perrin WF (2007) Generation length and percent mature estimates for IUCN assessments of cetaceans. Administrative Report, National Marine Fisheries Service, Southwest Fisheries Science Center, La Jolla, CA

Turvey ST, Pitman RL, Taylor BL, Barlow J and others (2007) First human-caused extinction of a cetacean species? Biol Lett 3:537-540

Wade PR (1998) Calculating thresholds to the humancaused mortality of cetaceans and pinnipeds. Mar Mamm Sci 14:1-37

Wang JY (2009) Stock identity. In: Perrin WF, Wursing B, Thewissen JGM (eds) Encyclopedia of marine mammals. Elsevier, New York, NY, p 1115-1118

> Wang JY, Hung SK, Yang SC (2004) Records of Indo-Pacific humpback dolphins, Sousa chinensis (Osbeck, 1765), from the waters of western Taiwan. Aquat Mamm 30:189-196

> Wang JY, Yang SC, Hung SK, Jefferson TA (2007a) Distribution, abundance and conservation status of the eastern Taiwan Strait population of Indo-Pacific humpback dolphins, Sousa chinensis. Mammalia 71:157-165

Wang JY, Yang SC, Reeves RR (2007b) Report of the Second International Workshop on conservation and research needs of the Eastern Taiwan Strait population of IndoPacific humpback dolphins, Sousa chinensis. National Museum of Marine Biology and Aquarium, Checheng

- Wang JY, Hung SK, Yang SC, Jefferson TA, Secchi ER (2008) Population differences in the pigmentation of Indo-Pacific humpback dolphins, Sousa chinensis, in Chinese waters. Mammalia 72:302-308

> Wang JY, Yang SC, Fruet PF, Daura-Jorge FG, Secchi ER (2012) Mark-recapture analysis of the critically endangered eastern Taiwan Strait population of Indo-Pacific humpback dolphins (Sousa chinensis): implications for conservation. Bull Mar Sci 88:885-902 